\title{
Transient global amnesia: current perspectives
}

This article was published in the following Dove Press journal:

Neuropsychiatric Disease and Treatment

24 October 2017

Number of times this article has been viewed

\author{
David R Spiegel \\ Justin Smith \\ Ryan R Wade \\ Nithya Cherukuru \\ Aneel Ursani \\ Yuliya Dobruskina \\ Taylor Crist \\ Robert F Busch \\ Rahim M Dhanani \\ Nicholas Dreyer \\ Department of Psychiatry and \\ Behavioral Sciences, Eastern Virginia \\ Medical School, Norfolk, VA, USA
}

Correspondence: David R Spiegel

Department of Psychiatry and Behavioral Sciences, Eastern Virginia Medical School, 825 Fairfax Avenue, Norfolk, VA 23507, USA

Email spiegedr@evms.edu

\begin{abstract}
Transient global amnesia (TGA) is a clinical syndrome characterized by the sudden onset of an extraordinarily large reduction of anterograde and a somewhat milder reduction of retrograde episodic long-term memory. Additionally, executive functions are described as diminished. Although it is suggested that various factors, such as migraine, focal ischemia, venous flow abnormalities, and epileptic phenomena, are involved in the pathophysiology and differential diagnosis of TGA, the factors triggering the emergence of these lesions are still elusive. Recent data suggest that the vulnerability of CA1 neurons to metabolic stress plays a pivotal part in the pathophysiological cascade, leading to an impairment of hippocampal function during TGA. In this review, we discuss clinical aspects, new imaging findings, and recent clinical-epidemiological data with regard to the phenotype, functional anatomy, and putative cellular mechanisms of TGA.
\end{abstract}

Keywords: transient global amnesia, vascular, migraines, psychiatric

\section{Introduction}

More than 50 years after its initial description, transient global amnesia (TGA) remains one of the most enigmatic syndromes in clinical neurology. TGA is defined as a sudden onset of an anterograde and retrograde amnesia that lasts up to 24 hours, although mild subclinical neuropsychological deficits with concomitant vegetative symptoms can last for days after the episode. The memory impairment of patients with acute TGA shows a profound reduction of anterograde and a milder reduction of retrograde episodic memory, including executive functions and recognition. In this review of TGA, we summarize the epidemiology, symptomatology, pathophysiology, assessment, differential diagnosis, longer-term outcome, and possible treatment recommendations associated with this condition. ${ }^{1}$

\section{Literature review}

Full-text articles and abstracts were selected for this review and were identified through searches of PubMed with the search terms "transient amnesia", "transient global amnesia", "hippocampus", and "amnestic syndromes" between 1985 and 2017. Additionally, more distant seminal articles on this subject matter were included. Only articles published in English were reviewed. The final reference list was chosen on the basis of relevance to the topics covered in this review (eg, their originality, contribution to hippocampal and TGA epidemiology, anatomy and physiology, pathophysiology, diagnosis, and treatment).

\section{Epidemiology}

The following prospective and retrospective systematic studies identify TGA cases from either hospitalized or national health registry samples. 


\section{Incidence}

Recent epidemiological data put the annual incidence of TGA between 3.4 and 10.4/100,000., ${ }^{2,3}$

\section{Age and gender}

TGA most commonly presents in the seventh decade of life. Across studies, the mean age of an episode ranges from 61 to 67.3 years. ${ }^{2,4-6}$ In a 2006 study, $96 \%$ of subjects with TGA $(n=142)$ presented between the ages of 51 and 80 years. Epidemiological studies fail to identify any subjects under the age of 55 years. Several small-scale studies show a slight female predominance. ${ }^{2}$ However, a study on 5,097 TGA cases shows gender distribution to be $50.7 \%$ females and $49.3 \%$ males, ${ }^{4}$ consistent with one previous analysis. ${ }^{5}$

\section{Recurrence}

Depending on the length of follow-up, the annual rate of TGA recurrence varies from 2.9 to $26.3 \% .{ }^{5}$ A 2005 study with a 7-year follow-up period, which is the longest systematic follow-up period reported, found a recurrence rate of $8 \%{ }^{6}$ Another study that recruited subjects over a 10 -year period ( $n=142$ ), however, with no designated follow-up period after the first occurrence, found a recurrence rate of $6.3 \%$, if probable episodes were taken into account. ${ }^{5}$

\section{Risk factors}

A migraine history is one of the more notable risk factors associated with developing TGA. In a 2014 population-based study $(n=316,602)$, migraine patients were significantly more likely to develop TGA than their matched controls, with the incidence rate ratio of $2.48 .^{7}$ Additionally, of the subjects who developed TGA after the age of 40 years, those with a history of migraine had a significantly younger age of onset (56.6) compared to the control group (61.4). No associations were found between various migraine subtypes and TGA. ${ }^{7}$

Cardiovascular risk factors are also well studied in TGA. A retrospective case-control study found age- and sex-matched control subjects ( $\mathrm{n}=293)$ to have significantly decreased odds of having hyperlipidemia and ischemic heart disease when compared with those subjects with TGA $(n=293)$. Within this same study, 632 transient ischemic attack (TIA) subjects had greater rates of hypertension, diabetes mellitus, ischemic stroke, and atrial fibrillation when compared with TGA subjects, likely indicating differing risk factors between TGA and TIA. ${ }^{8}$ Furthermore, a retrospective study of 85 TGA subjects revealed that those with history of two episodes of TGA showed a higher frequency of carotid atheromasia and ischemic heart disease than those with a history of just one episode of TGA. ${ }^{9}$ Also, of note is that cancer diagnosis carries no increased risk of TGA, according to a prospective cohort study with 5,365,608 subjects running between 2001 and 2009. ${ }^{4}$

\section{Psychiatric comorbidity}

One study compared psychiatric disease in 51 subjects who experienced a TGA to 51 subjects who experienced a TIA. Psychiatric disease was defined as having "a diagnosis of depression or anxiety disorder" or having received "treatment with specific drugs for at least 3 months". TGA subjects had a significantly higher percentage of psychiatric disease compared to TIA controls (39.2\% vs $13.7 \%$, age- and sex-adjusted odds ratio $[\mathrm{OR}]=2.86) .{ }^{6}$ Additionally, a significantly higher percentage of TGA subjects (33.3\%) reported a family history of psychiatric disease as compared with TIA subjects (13.7\%).

In summary, according to community-based studies, the annual incidence of TGA is $5-10 / 100,000$ and $23.5-$ $32 / 100,000$ for people aged 50 years and older. Peak incidence is around the age of 62 years (standard deviation [SD] 10 years). A total of $54 \%-67 \%$ of TGA patients are female. Chances of recurrence are reported variedly from 2.9 to $25 \%$. Other than migraine headaches, there are no definitive risk factors for the development of TGA. ${ }^{10}$

\section{Clinical diagnosis Development of TGA criteria}

TGA is a clinical diagnosis. It was first described in 1956 as an "isolated episode of confusion with amnesia" not otherwise associated with other neurological deficits. ${ }^{11,12}$ Subjects were described as becoming repetitious and asking the same questions, although mostly revolved around the memory loss itself. Fisher and Adams ${ }^{13}$ coined the term TGA in 1958; however, it was not until 1964 that they detailed a report of 17 patients with sudden onset anterograde amnesia and confusion that resolved within a few hours. ${ }^{14}$

Hodges and Warlow ${ }^{15}$ later developed criteria for the clinical syndrome in 1990 (Table 1), and since then, this has been used as the foundation of TGA diagnosis. They

Table I Hodges and Warlow criteria for TGA

Diagnostic criteria of TGA

- Attacks must be witnessed

- There must be anterograde amnesia during the attack

- Cognitive impairment is limited to amnesia

- No clouding of consciousness or loss of personal identity

- No focal neurological signs/symptoms

- No epileptic features

- Attack must resolve within 24 hours

- No recent head injury or active epilepsy

Note: Data from Hodges and Warlow. ${ }^{15}$

Abbreviation: TGA, transient global amnesia. 
divided patients into the following three categories: pure TGA, probably epileptic amnesia, and probably TIA. Exclusionary criteria for pure TGA included focal neurological symptoms, such as ataxia, limb weakness, and sensory disturbances. Of the 153 patients reviewed between 1984 and 1987, 114 patients met the proposed criteria for pure TGA. The majority of attacks in this group lasted between 1 and 8 hours. Both TGA and non-TGA groups showed disorientation in time and repetitive questioning, although the TGA group demonstrated more repetitive questioning with $92 \%$ vs $71 \%$ in non-TGA groups. Most patients with TGA had a permanent retrograde amnesic gap for the events immediately prior to and during the attack, although this was also seen in non-TGA cases. ${ }^{15}$

\section{Precipitating events}

A study of 142 cases of TGA found precipitating factors in 131 of these episodes $(89.11 \%) .{ }^{5}$ Emotional stress (ie, triggered by gastric endoscopy, birth/death announcement, and difficult/exhausting work day), physical effort (ie, gardening, house work, and sawing wood), and water contact/ temperature change (ie, hot bath/shower and cold swim) were observed most frequently immediately before an attack and are considered "close events". Anxiety triggered by conflict at home or work, health problems, and financial stressors were often reported weeks prior to TGA and are considered "remote events". In the TGA series, the percentage of emotional stress, physical effort, and water contact/temperature change was 29,25 , and $14 \%$, respectively, compared to 48,9 , and $0 \%$ in control groups, respectively. Remote events of anxiety and exhaustion were reported in 24 and 33\% of TGA cases and 6 and 90\% in controls, respectively. Differences from control subjects in both close $(P<0.000)$ and remote precipitating events were found to be statistically significant, providing strong evidence that TGA occurs in certain contexts.

\section{Associated symptoms}

Although focal neurological deficits exclude a diagnosis of TGA, there are several nonfocal symptoms, which are often observed. Headache and nausea/vomiting are the most common and were each present in $10 \%$ of TGA cases immediately after the attack. ${ }^{15}$ Dizziness, chills or hot flushes, fear of dying, cold extremities, paresthesias, emotionalism, trembling, chest pain, and sweating have also been reported. ${ }^{5}$ Quinette et al proposed that such associated symptoms were somatic manifestations of anxiety and found that, compared to controls, TGA episodes were more frequently related to the Diagnostic and Statistical Manual of Mental
Disorders, fourth edition criteria of panic attacks. While these symptoms have been useful in identifying areas of dysfunction, TGA diagnosis should be made based on criteria listed in Table 1.

In summary, while multiple exclusionary criteria exist, heteroanamnestic confirmed anterograde amnesia in a clear sensorium and cognitive impairment limited to amnesia is the main "rule-in" criteria for TGA. Additionally, the amnesia must last $<24$ hours. Headache, dizziness, and nausea are the most common accompanying complaints. Finally, in $89 \%$ of cases, some provoking activity can be pointed out immediately before the attack occurred. Physical exertion (including sexual activity) is the most common precipitating event, followed by emotional stress and sudden change of temperature. ${ }^{10}$

\section{Pathophysiology Relevant neuroanatomy}

The clinical picture of TGA has led researchers to investigate focal injury to the neurological circuits involved in memory as a potential etiology of the syndrome. Hodges and Warlow ${ }^{15}$ performed computed tomography (CT) scans on $83 \%$ of the pure TGA cases in their study ( $\mathrm{n}=95$ ), finding small white matter changes, basal ganglia lesions, or periventricular lucencies in $12 \%$ of these patients $(n=11)$. These areas, however, are not located in known memory-related structures. Current evidence points to the formation of early memory within the hippocampal/entorhinal cortex network and its eventual transference to remote memories stored in the neocortex network. ${ }^{16}$ Particular attention has been given to the cornu ammonis (CA1) field of the hippocampus, ${ }^{17}$ which has been hypothesized to play a central role in the pathophysiology of TGA given its extraordinary sensitivity to cell stress. ${ }^{18}$

More recent studies have found imaging changes strongly related to TGA using magnetic resonance diffusion-weighted imaging (DWI). These findings are typically unilateral and tend to be small (1-3 mm), high-signal foci found in the CA1 field of the hippocampus. ${ }^{17-20}$ These lesions are most prominent 24-48 hours after the initial TGA episode, ${ }^{21}$ which matches the peak activation period for microglia in stroke models..$^{22}$ Alternatively, there are cases of TGA with bilateral and even multifocal hippocampal involvement and also cases with ischemic or hemorrhagic damage to other brain regions. ${ }^{22-27}$ These conflicting reports do not help to clarify the initial neuronal insult in TGA.

Similarly, the underlying etiology of TGA remains obscure with multiple proposed mechanisms, such as arterial ischemia, venous congestion, migraine, and psychogenic 
disorders. Early researchers hypothesized that atherosclerotic or thromboembolic events disrupt blood flow to the hippocampus, which is supplied by the posterior cerebral artery and the hippocampal arteries. ${ }^{20,23,24}$ Venous congestion and jugular vein valve insufficiency are also hypothesized etiologies, given that many patients report Valsalva-associated maneuvers prior to a TGA event. ${ }^{19,28}$ In addition, severe emotional reactions and migraines may contribute to the destabilization of the CA1 sector of the hippocampus via massive glutamate release. ${ }^{28}$ The following sections explore these postulated mechanisms in greater detail.

\section{Arterial ischemia and venous congestion}

There are many parallels between TGA and TIA, which support the arterial ischemia hypothesis. TGA and TIA are characterized by an abrupt onset of reversible loss of function occurring in patients within similar age group demographics. Reported mean age of onset is 60-66 years for TGA and 69-71 years for TIA, with modal onset, for both, over the age of 50 years. ${ }^{5}$ Despite these similarities, statistical analysis has reliably shown significantly reduced atherosclerotic risk factor profiles, including decreased prevalence of embolic heart disease, diabetes mellitus, hypertension, and carotid artery atherosclerotic disease, in patients with TGA in comparison to those with TIA. ${ }^{6}$ Population cohort data have also consistently indicated that a history of TGA does not put patients at a higher risk of cerebrovascular events, and in fact, recent studies suggest a more favorable prognosis for TGA patients compared to TIA patients in regard to risk of future cerebrovascular events. ${ }^{8}$ Even more, one retrospective analysis showed no heightened risk of future cerebrovascular event following a TGA event when compared with healthy matched controls, further refuting an atherosclerotic hypothesis. ${ }^{8}$

Several prominent clinical features of TGA additionally argue against arterial ischemia as a likely mechanism. The mean duration of a TGA episode largely exceeds that seen in TIA, with a documented mean duration of 4-8 hours and $>97 \%$ of episodes lasting $>1$ hour. ${ }^{5}$ For TIA, in contrast, the vast majority of episodes last $<60$ minutes, with the bulk of these lasting only a few minutes. ${ }^{29}$ Also, the absence of associated focal neurologic dysfunction during the TGA episode, such as lateralizing weakness and visual field deficits, is inconsistent with the ischemic hypothesis, which would be expected in cases of an acute ischemic event involving bordering neuroanatomy.

The pathophysiology behind frequently cited precipitating events and comorbidities accompanying TGA (including
Valsalva-like activities, anxious and phobic personality traits, emotional stressors, and immersion in hot or cold water) has supported an arterial mechanism. ${ }^{5,30}$ It has been postulated that an increased prevalence of patent foramen ovale among patients with TGA is responsible for its association with Valsalva-like activity by means of paradoxical embolism; however, further attempts to reproduce this association have since failed. ${ }^{31,32}$ Increased incidence of TGA among particular personality traits and precipitation by emotional stressors suggest that reactive hyperventilationinduced cerebral vasoconstriction can result in changes in the cerebrovascular hemodynamics within the hippocampal region. ${ }^{20}$ Functional imaging technology supports such reactive changes, demonstrating relative hypoperfusion within the medial temporal lobes (MTLs) in patients with TGA. ${ }^{33}$ Results among other studies, however, have been inconsistent, and it is unclear whether the findings are causative in nature or reactive to derangements in cerebral metabolism. ${ }^{33,34}$

Structural magnetic resonance imaging (MRI) also provides support for an ischemic mechanism, with DWIMRI showing evidence of abnormal lateral hippocampal punctate hyperintensities in patients with TGA. Analysis of the appearance and evolution of these lesions over time have displayed similarities to previously described lesions from cerebral ischemic injury, with reported detection rates ranging from 57 to $100 \%$ in patients with TGA. ${ }^{19,35-38}$ These lesions primarily involve the hippocampal CA1 neuronal field, a region known to be critically involved in the process of memory consolidation and to be vulnerable to stress.

However, several temporal and anatomical aspects of these lesions are fundamentally inconsistent with conventional ischemic lesions. Lesions associated with TGA were most reliably seen 24-72 hours after symptom onset and were shown to disappear soon after. Additionally, the typical duration of TGA episodes (4-6 hours) is inconsistent with this delayed and reversible nature of DWI-MRI lesions, which when present in the case of TIA are detected much earlier and persist longer. ${ }^{39}$

Venous congestion with retrograde cerebral flow is another prominent hypothesis for the pathophysiology behind TGA. ${ }^{30}$ Valsalva-like activity causes transient elevation of intrathoracic pressure with obstruction of venous return, potentially resulting in retrograde transmission of pressure to the cerebral venous vasculature draining the involved structures. Other frequently cited precipitating events (cold water immersion, exercise, and emotional stressors) are mechanistically analogous by the way of increasing sympathetic tone 
with central diversion of peripheral venous volume in effort to preserve flow to vital organs, which similarly results in elevated central venous pressures. ${ }^{39}$ Proponents also recognize the anatomy of cerebral venous drainage as supportive evidence, as venous outflow from bilateral hippocampal regions converge to a common great vein of Galen before later diverging at the confluence of venous sinuses supporting perturbation of bilateral hippocampal function previously unexplained by ischemic hypotheses. ${ }^{40}$

Numerous studies have demonstrated a significant increase in the prevalence of jugular venous valve insufficiency with jugular vein retrograde flow among TGA patients when compared with healthy matched controls. ${ }^{40-42}$ This association was detected via MRI and Doppler and was particularly common among patients reporting a concomitant precipitating event. The largest study demonstrates $80 \%$ prevalence of internal jugular venous valve insufficiency with retrograde flow among 142 TGA subjects compared to only $25 \%$ among control subjects. ${ }^{41}$ However, several recent studies have brought the significance of this finding into question. One study using transcranial Doppler sonography of intracranial vessels to record blood flow direction and velocity at the internal jugular veins, basal veins of Rosenthal, and great vein of Galen compared findings at rest and during Valsalva-like maneuvers. Although confirming an elevated prevalence of jugular valve insufficiency among TGA patients, intracranial venous reflux was not seen in TGA or in control subjects. ${ }^{43}$ This was confirmed by a study utilizing time of flight magnetic resonance angiography to observe abnormal jugular venous reflux within TGA and control patients but similarly found low rates in each study group (intracranial retrograde flow in 7/167 in TGA group, 8/167 in emergency room visitor control group, and 3/167 in healthy matched control group). ${ }^{48}$

In summary, although the physiological findings suggest a meaningful association with TGA, there is limited evidence for venous congestion as an etiology for TGA. Current evidence is unable to explain the lack of association with other causes of venous congestion, including congestive heart failure and cerebral venous thrombosis. ${ }^{18}$ It is also unclear why such transient pressures can induce the long-lasting effects seen in TGA. Further questions surround the true association with reported precipitating events, including why episode recurrence is so uncommon when they can be prompted by a Valsalva-like activity. It is likely that vascular mechanisms play a role in the pathophysiology of TGA, but the exact role remains to be discovered.

\section{Migraine}

Another hypothesis is that TGA may be a sequela of migraines due to sweeping depression of cerebral activity that is found throughout the cortex, extending through the hippocampus, leading to transient dysfunction and subsequently TGA. ${ }^{45}$ It is this same cortical depression that is thought to give rise to the aura found in migraine sufferers and is caused by the release of massive glutamate and a subsequent wave of short-lasting cortical depolarization. ${ }^{46}$

This mechanism has been demonstrated in animal models by local stimulation of the hippocampus, lending to the possibility that a similar reaction could be elicited in humans via the experience of strong emotional events or other intense stimuli leading to a large release of glutamate from hippocampus. ${ }^{47}$ Given this relationship between migraines and transient hippocampal dysfunction, it is feasible to consider the possibility of an etiological relationship between migraines and the transient memory problems exhibited by patients with TGA. However, there is a dearth of evidence to support this theory. Currently, the evidence supportive of a connection between migraines and TGA is mainly associative and causality cannot be stated with any significant level of confidence given the observational nature of these studies. ${ }^{5,28}$

\section{Psychogenic causes}

One of the lesser studied etiologies of TGA is of psychogenic origin. In these cases, the precipitating trigger is often an emotional event or psychological stressor. It is encountered classically in younger populations and is often associated with a subjective indifference to the memory loss experienced. ${ }^{45}$ In most cases, autobiographical memory deficits, which are most often intact in TGA, are appreciated with relatively functioning anterograde memory formation capabilities. Physiologically, the insult appears to disrupt the affective learning circuit formed between amygdala, hippocampus, striatum, and prefrontal cortex. TGA could be viewed as an illness of temporary hippocampal insufficiency, where its inhibitory effects to the amygdala are disrupted, which could precipitate a disruption in memory formation. ${ }^{28}$

Other evidence suggests that approximately half of the patients with psychological disturbances as the precipitating etiology of TGA also had associated phobic personality trait. ${ }^{28}$

Psychogenic amnesia can be linked to several psychiatric disorders including posttraumatic stress disorder and dissociative disorders, where the loss of memory could be considered a defensive psychological mechanism. ${ }^{28}$ 


\section{Summary of pathophysiology}

There are several potential etiologies that may be responsible for TGA, including arterial ischemia, venous congestion, migraine, and psychogenic disorders. It is possible that the mechanisms described earlier are associated with TGA by which they are involved in a common pathway that ultimately destabilizes the CA1 region of the hippocampus. At this time, discovery of a specific cause may not change the course of management or the outcome, as the nature of the disease process is self-limiting.

\section{Differential diagnosis}

The differential diagnosis of TGA includes those disease states that can present with transient anterograde amnesia (Table 2). Other causes of such disease states are ruled out prior to reaching the diagnosis of TGA (Figure 1). ${ }^{110,111}$

\section{Transient epileptic amnesia (TEA)}

TEA is a form of adult-onset MTL epilepsy that presents as recurrent, transient episodes of acute memory loss. ${ }^{7,48-50}$ This amnesia may be antero-retrograde or only retrograde. ${ }^{49}$ This is distinct from TGA, in which patients always have anterograde memory loss and may have permanent retrograde amnesia that spans hours to days prior to the onset of the episode. ${ }^{45}$ Patients with TGA are more likely to exhibit agitation or anxiety. ${ }^{50}$ While some authors report repetitive questioning as a feature indicative of TGA, others have found that TEA patients also exhibit this behavior. ${ }^{48,49,52}$

Important features of TEA that differ from TGA include occurrence upon awakening, duration $<1$ hour, interictal retrograde amnesia incomplete anterograde amnesia (able to remember not being able to remember), temporal lobe features such as olfactory/gustatory hallucinations, oral automatisms, and interictal EEG abnormalities. ${ }^{7,48,53}$ Controversy exists over abnormal EEG being a diagnostic factor of TEA, as only a third are found to have abnormalities, while the other twothirds show focal slowing or normal findings. ${ }^{5,44,48}$ Subsequent follow-up reveals that TEA has a higher recurrence rate than TGA..$^{5,44,48}$ The diagnosis of TEA carries a favorable prognostic response to antiepileptic drugs ${ }^{49,52,53}$ but may yield persisting interictal memory deficits. ${ }^{53,54}$ This includes accelerated long-term forgetting and autobiographical amnesia. ${ }^{54}$

\section{Ischemic events}

Ischemic events such as cerebrovascular accidents (CVAs) and TIAs must be excluded prior to arriving at a diagnosis of TGA, as the former typically requires emergent treatment. CVAs and TIAs are most commonly associated with focal neurological deficits, which by definition preclude TGA from the working diagnosis. However, to confound matters, unilateral, isolated hemispheric infarction of the hippocampus or thalamus may present with amnesia as the sole manifestation of the CVA, thus becoming difficult to differentiate from TGA. ${ }^{55,56}$ Similarly, ischemia within the MTL with involvement of the hippocampus, caudate, or fornix has also been associated with a TGA-like presentation. ${ }^{25,57-60}$ Neuroimaging is strongly recommended. For this reason, neuroimaging is strongly recommended in atypical presentations of TGA or unwitnessed episodes, due to the risk of head trauma or CVA. ${ }^{43,61,62}$ While TGA does not typically show acute changes on brain imaging, MRI with DWI or T2-weighted imaging may reveal hyperintense punctate lesions in the lateral hippocampal regions in the subacute phase $\sim 48-72$ hours after the onset of TGA symptoms. These lesions are visible for up to 7-10 days after onset. ${ }^{17,19,36,61,62}$

Brain MRI is usually performed to exclude stroke at the time of symptoms; however, this may be too early to discover the findings associated with TGA. Additionally, typical risk factors associated with cerebrovascular disease should be assessed in the patient's history. The likelihood of CVA/ TIA as the etiology of transient anterograde amnesia may be increased with the presence of these risk factors. ${ }^{61,62}$

\section{Migraines}

Migraine is an episodic headache disorder accompanied by various neurological, gastrointestinal, and autonomic changes. In those with migraine headaches, $\sim 20 \%$ experience an aura, either during or before the development of the headache. ${ }^{63}$ Similar to glutamate-mediated transient neuronal depolarization followed by prolonged quiescence of neuronal activity, CSD may be explained by hypoperfusion that is preceded by transient hyperperfusion in the cortex. ${ }^{18} \mathrm{CSD}$ has been proposed as the neurophysiological explanation of migraine with auras, as it propagates at a similar velocity as visual scotomata during an aura. ${ }^{63}$

There are multiple case reports suggesting that TGA occurs as either an aura to or, "associated with", migraine headaches. ${ }^{64-69}$ Otherwise, in two larger scale studies, the association between TGA and migraines is mixed. For instance, in an 11-year retrospective study reported in 2015, among 8,821 new migraine patients, six cases of TGA were identified during a migraine attack. For a majority of these patients, TGA occurred after the beginning of the attack. ${ }^{51}$ Alternatively, in a 1998 case-control study, TGA patients with migraine were identified from a group of 57 TGA patients. The former group was then compared with 


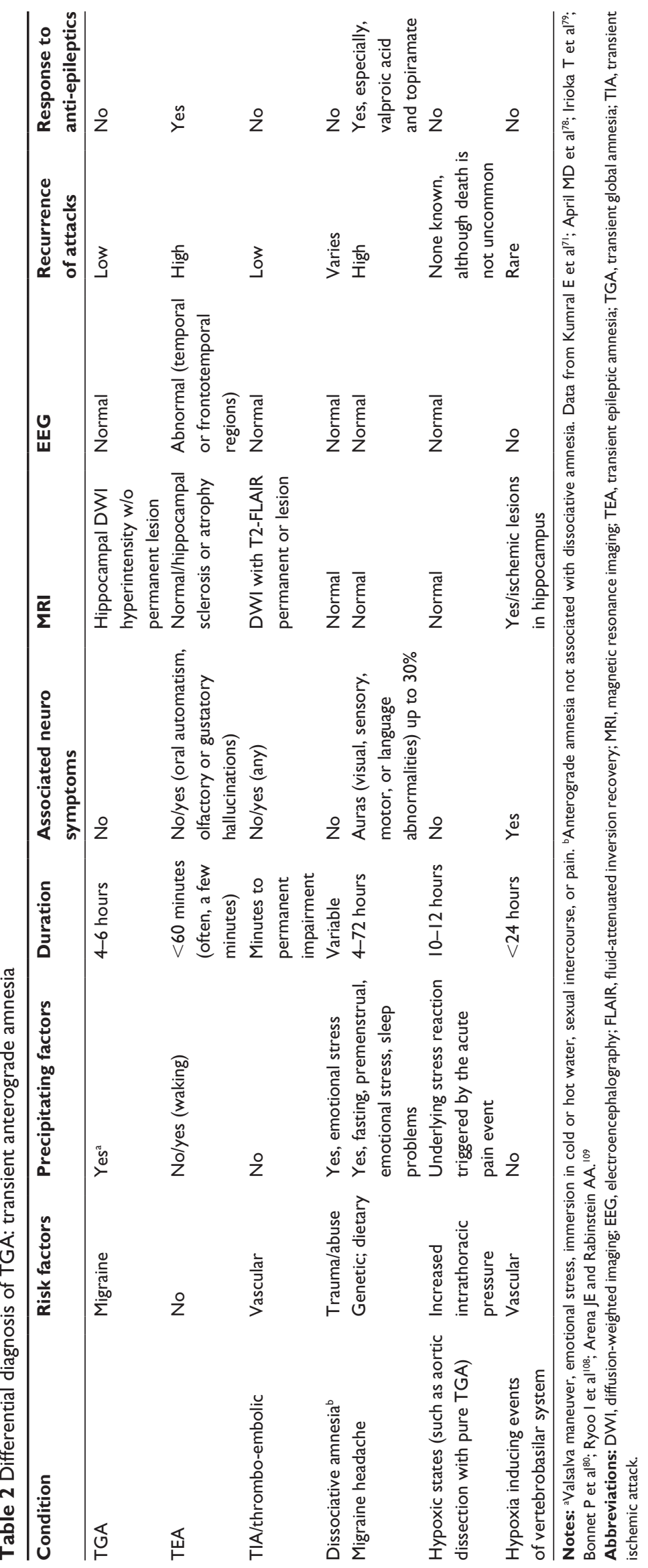




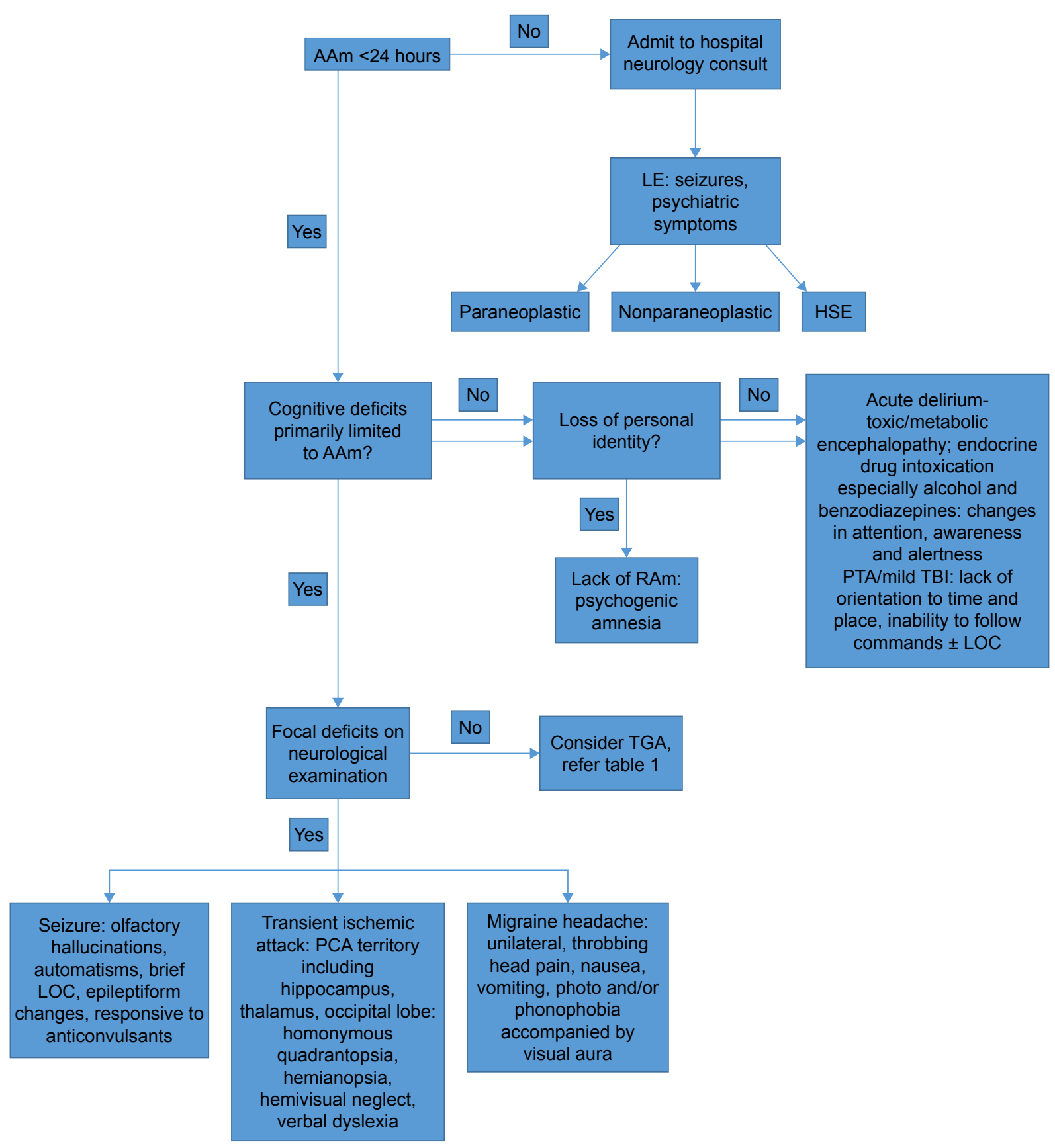

Figure I Diagnostic algorithm of anterograde amnesia.

Abbreviations: AAm, anterograde amnesia; EEG, electroencephalogram; HSE, herpes simplex encephalitis; LE, limbic encephalitis; LOC, loss of consciousness; PCA, posterior cerebral artery; PTA, posttraumatic amnesia; RAm, retrograde amnesia; TBI, traumatic brain injury.

two groups of patients, one with TGA only and the other with normal controls. Despite the prevalence of both migraine and tension headaches being increased in those patients with TGA, there was no evidence of an increased frequency in TGA features in those with migraines. ${ }^{70}$

\section{Hypoxia}

In addition to ischemia in the setting of TIA/CVA, hypoxiainducing events with vasospastic, vasoconstrictive, or arterial insufficiency etiologies have been described in the literature as causes of TGA-like symptoms. For instance, cardioembolism and large-artery disease of the vertebrobasilar system can lead to hippocampal infarcts. Five variants have been described including anterior, posterior, unilateral complete, bilateral, and small circumscribed (punctiform) hippocampal infarcts. Prominent clinical acute anterograde amnesia and retrograde amnesia were present in more than two-thirds of patients. In most of the patients, the lesions 
involved the anterior and medial aspects of the hippocampus along the complete length of the head, body, and tail of the hippocampus. ${ }^{71}$ Such evidence supports that TGA's etiology may be a compromised vertebrobasilar system leading to the dysfunction of MTL and subsequently memory formation.

Several case reports describe TGA-like symptoms after vascular procedures or intravenous contrast use..$^{51,71-75}$ Contrast media are sterile iodine-containing solutions used in diagnostic imaging procedures. Older agents generally fall into the class of ionic monomers, which have a high osmolality and a high chemotoxicity. Nonionic agents have been developed to overcome the adverse events associated with older contrast media. While there have been cases describing nonionic angiographic contrast medium neurotoxicity, the risk of neurotoxicity remains higher in the ionic class of contrast media. Furthermore, it has been suggested that the risk of neurotoxic effects might be increased by preexisting abnormalities of the blood-brain barrier and by repeated contrast injections.

Other postulated mechanisms by which cerebral angiography produces TGA symptoms include epilepsy and ischemia. $^{73,74}$

Other case reports support an arterial insufficiency etiology, describing TGA symptomatology secondary to vascular disease processes. ${ }^{76-80}$ Intriguingly, aortic dissection, a potentially critical break in the lining of the main arterial outflow from the heart classically associated with severe chest pain and frequently a devastating diagnosis, has been reported to present with TGA-like symptoms in nine cases, five of whom were without chest pain, ${ }^{78}$ although the latter could be attributed to the patients' altered mental status. Regardless, recognition of painless aortic dissection in the differential of TGA is imperative. While the latter is generally a benign syndrome, the former could result in significant morbidity or mortality. Thus, in those patients presenting with TGA-like symptoms and accompanying cardiovascular changes such as hypotension and hypertension or asymmetric extremity blood pressures, CT angiography of the abdomen may be warranted..$^{81}$

Finally, others report the development of TGA after use of phosphodiesterase type 5 inhibitor and sexual activity. However, it is unclear whether the phosphodiesterase inhibitor or the sexual activity caused the vasospasm, as both are known to involve significant changes in blood flow. ${ }^{82}$

\section{Psychogenic amnesia/dissociative amnesia}

Psychogenic amnesia, often labeled as dissociative amnesia, is differentiated from TGA, in which the former affects retrograde memory, it is generally episodic, and it is often accompanied by intense psychological distress. While often times complicated medical disorders become labeled as "psychogenic" if the etiology is unknown, there is literature that supports a psychogenic origin to TGA. ${ }^{83}$

Data gathered from two geriatric studies utilized the Geriatric Depression Scale in patients with TGA, showing that $40 \%$ of TGA patients had depressive symptoms. ${ }^{84}$ A more detailed study investigated phobic personality traits of 51 TGA patients and established that $82 \%$ had pathological avoidance behaviors. ${ }^{83}$

TGA patients have a high prevalence of comorbid emotional distress and anxious personality traits. ${ }^{5,85}$ About one-third of TGA episodes occur after physical or psychological stress, which suggest disruption of memory formation due either to ischemic or stress-induced catecholamine disruption. ${ }^{86,87}$ Some authors believe that stress-induced catecholamine release may lead to hypoxia or ischemia, whereas others believe that the neurotransmitters involved may affect the formation of memory. ${ }^{88-93}$ There is some evidence of an association between TGA and Takotsubo syndrome. The latter is characterized by transient, acute left-ventricular myocardial dysfunction mimicking myocardial infarction that also occurs after physical or psychological stress. ${ }^{88}$

If the clinical picture remains unclear, neuropsychological testing or other neurological tests (electroencephalogram and neurological examination) may be helpful in distinguishing psychogenic amnestic disturbances from neurological amnestic conditions, especially in patients who have secondary gain or overembellish symptoms. ${ }^{28}$

In summary, to make a diagnosis of TGA, all strict clinical criteria as shown in Table 1 should be met. The differential diagnosis includes structural (vascular) disease, epileptic amnesia, delirium, intoxication, and head injury and migraine headaches. When a general physician is presented with a typical case, no additional diagnostic tests, such as MRI and EEG, are needed. This even holds when such a patient has vascular risk factors. However, when focal neurological signs accompany anterograde amnesia, neuroimaging is warranted and potentially a neurology consult. If retrograde amnesia is also affected and emotional distress is present, the clinician needs to consider psychogenic amnesia. Alternatively, albeit rare but more ominous, are those cases of "painless" aortic dissection, where TGA is the primary symptom as chest pain is lacking. Finally, research findings using DWI-MRI support the concept of focal ischemia. These focal lesions have a maximum detectability between 48 and 72 hours after the start of TGA. Focal lesions have been demonstrated in the 
CA1 area of the cornu ammonis of the hippocampus (MTL), mostly unilateral. However, in up to $35 \%$ of TGA patients, no such abnormalities are demonstrable. ${ }^{10}$

\section{Beyond 24 hours: outcomes}

According to the standard accepted criteria, deficits of TGA resolve within 24 hours. ${ }^{15}$ There has been considerable research dedicated to the cognitive profile of TGA patients following the resolution of acute-phase symptoms; however, results have been mixed.

A 2009 meta-analysis examined data from 25 different studies and compared 374 TGA subjects to 760 control subjects in the following five domains: anterograde episode long-term memory, retrograde episode long-term memory, short-term memory, semantic memory, and executive function. Beyond the first 24 hours, there was no significant difference between patients and controls in any of the five domains for the 30-day period following TGA onset. ${ }^{94}$ Similarly, a study with a median follow-up period of 1,128 days found no significant difference in performance between TGA patients and controls on tasks of episodic memory, semantic memory, working memory, executive functions, and attention. ${ }^{95}$ Alternatively, one study with follow-up periods of 4 months and 1 year found deficits in anterograde memory in TGA subjects when compared with controls; however, only when data from both follow-up periods were pooled together. Additionally, at the 4-month follow-up visit, higher scores on anxiety and depression scales correlated with worse performance on tests of retrograde and anterograde memory tests, respectively. ${ }^{96}$

Given the cardiovascular hypothesis of TGA and the interest in the field in cardiovascular risk factors of the disease, one study examined the subsequent risk of experiencing CVA in patients with a history of TGA vis-a-vis comparing the rate of CVA following TGA to the rate of CVA following migraine, seizure, and TIA at time points of 1 and 5 years after the event. The results of this study did not support an increased risk of CVA after TGA, with the risk of CVA at 1 year being similar to that after migraine $(0.54$ and $0.22 \%$, respectively) and lower than after TIA (4.54\%). The 5-year rates for CVA following TGA, migraine, and TIA were $2.44,0.86$, and $12.23 \%$, respectively. ${ }^{97}$

In a 12-year follow-up study, the longest in the literature to date, TGA patients were compared with controls on end points of CVA/TIA, seizures, and cognitive impairment (mild cognitive impairment or dementia). TGA patients were not found to be at a higher risk of developing any of the included conditions than the control subjects. ${ }^{2}$

\section{Treatment}

By definition, TGA is a self-limited condition that resolves without intervention; thus, there is no specific treatment indicated. ${ }^{98}$ Although the mean time course of an amnestic episode in TGA is $4-6$ hours, with most resolving by 8 hours, proposed treatments could depend on uncovering the underlying etiology. ${ }^{5}$

If the presentation in TGA was secondary to reversible ischemia, as has been suggested by some case reports using DWI, as well as other reports that noted onset of TGA episodes after Valsalva-like maneuvers that may temporarily reduce cerebral blood flow, then optimization of cardiovascular factors similar to treatments implemented for cardiac ischemia, such as antiplatelets, managing blood pressure, and heart rate, or statin therapy could be beneficial. ${ }^{98-101}$

Some studies suggest an epileptic etiology of TGA, as electroencephalogram findings have been suggestive of epileptic discharges, but other case-control studies have found no such correlation. ${ }^{102-104}$ Thus, anticonvulsant medications would not be a proposed treatment option based on the available evidence.

Evidence suggesting that TGA is secondary to cortical depression following cortical hyperstimulation does not support theoretical treatment options with the exception of those related to migraine headaches. ${ }^{70}$ Triptans have not been studied in TGA.

Ultimately, there are no established, evidence-based treatments for TGA to date, likely due to the short duration of symptoms experienced as well as a lack of universally accepted pathophysiology.

\section{Conclusion/future directions}

The yearly incidence of TGA is $3-8$ cases per 100,000 people, although $6 \%-10 \%$ of patients with TGA will experience a second or third episode. ${ }^{105}$ Thus, while it may be difficult to predict an index episode of TGA, it may be possible to decrease the risk of future episodes. The latter could be accomplished through clinical trials by addressing different proposed etiologies associated with TGA. For instance, despite significantly reduced atherosclerotic risk factor profiles in patients with TGA in comparison to those with TIA, ie, diabetes mellitus and hypertension, can statins, antihypertensives, and/or hypoglycemics decrease the risk of arterial ischemia, which has been proposed to explain TGA? Can prophylactic treatment of migraines with triptans decrease the risk of TGA, as this is the only identified risk factor in the development of TGA in those under the age of 56 years? As discussed, TGA may be a sequela of migraines 
due to sweeping depression of cerebral activity. $N$-methyl-Daspartate (NMDA) receptors have a critical role in excitatory synaptic transmission, excitotoxicity, and plasticity in the central neuronal system. ${ }^{106}$

Both competitive and noncompetitive NMDA receptor blockers are able to modulate spreading depolarizations (SD). Phencyclidine (PCP), dizocilpine, and ketamine have shown the capacity to elevate SD electrical threshold, block or slow SD propagation, and reduce SD duration and speed. However, the use of PCP and dizocilpine is limited because of their harmful side effects. Recently, discovered neuroprotective, anti-inflammatory, and antitumor effects of ketamine have been reported. Given the findings of usefulness in lowdose regimens of ketamine, perhaps this noncompetitive antagonist of the NMDA receptor could decrease the risk of TGA $?^{107}$

Additionally, selective vulnerability of hippocampal CA1 neurons to metabolic stress seems to play a crucial part in the pathophysiological cascade that leads to a transient perturbation of memory pathways in TGA. Given this information, TGA could be classified as a "natural lesion model" of a perturbation of hippocampal CA1 neurons; further understanding of this pathogenesis could facilitate insights in other neurological disorders that affect the hippocampus, such as CVA, encephalitis, and Alzheimer's disease. ${ }^{18}$

Another remaining area of interest with regard to TGA is the discrepancy in timeline of brain imaging results and the transient nature of memory symptoms. More specifically, clinical/cognitive recovery from TGA over the course of a few hours is generally favorable. Recently, MR imaging studies have reported that small punctate high-signal intensity lesions are frequently found in the lateral portion of the hippocampus (ie, CA1 region) on DWI, with variable detection rates of the TGA lesions in the hippocampus on the first day of the symptom onset. The detection rates of the lesions on DWI rise with increased time lapse after the onset of symptoms and have been reported to be best detected 2-3 days after the episode. Several previous studies suggest that delayed neuronal injury of the hippocampus is a possible cause of the delayed appearance of the lesions. However, the small size of TGA lesions, being susceptible to partial volume averaging due to a relatively larger voxel size, could be the main cause for this delayed lesion appearance. Thus, utilizing high field strength could increase the lesion detectability on DWI. DWI protocol considering these factors, along with voxel size, can support a differential diagnosis in the emergency department and may help to understand the pathophysiological mechanisms of this curious disorder. ${ }^{108}$

\section{Disclosure}

Dr Spiegel is on the speaker's bureau for Allergen Pharmaceuticals. The authors report no other conflicts of interest in this work.

\section{References}

1. Sancesario G, Esposito Z, Mozzi AF, et al. Transient global amnesia: linked to a systemic disorder of amino acid catabolism? J Neurol. 2013;260(5):1429-1432.

2. Arena JE, Brown RD, Mandrekar J, Rabinstein AA. Long-term outcome in patients with transient global amnesia: a population-based study. Mayo Clin Proc. 2017;92(3):399-405.

3. Berli R, Hutter A, Waespe W, Bachli EB. Transient global amnesianot so rare after all. Swiss Med Wkly. 2009;139(19-20):288-292.

4. Zhu J, Lu D, Sveinsson O, et al. Is a cancer diagnosis associated with subsequent risk of transient global amnesia? PLoS One. 2015;10(4): e0122960.

5. Quinette P, Guillery-Girard B, Dayan J, et al. What does transient global amnesia really mean? Review of the literature and thorough study of 142 cases. Brain. 2006;129(pt 7):1640-1658.

6. Pantoni L, Bertini E, Lamassa M, Pracucci G, Inzitari D. Clinical features, risk factors, and prognosis in transient global amnesia: a follow-up study. Eur J Neurol. 2005;12(5):350-356.

7. Lin KH, Chen YT, Fuh JL, et al. Migraine is associated with a higher risk of transient global amnesia: a nationwide cohort study. Eur J Neurol. 2014;21(5):718-724.

8. Jang JW, Park SY, Hong JH, Park YH, Kim JE, Kim S. Different risk factor profiles between transient global amnesia and transient ischemic attack: a large case-control study. Eur Neurol. 2014;71(1-2): $19-24$.

9. Agosti C, Akkawi NM, Borroni B, Padovani A. Recurrency in transient global amnesia: a retrospective study. Eur J Neurol. 2006; 13(9):986-989.

10. Erkelens CD, Snoek JW. What doctors should not forget about transient global amnesia. Eur J Gen Pract. 2010;16(3):182-185.

11. Jaffe R, Bender MB. E.E.G. studies in the syndrome of isolated episodes of confusion with amnesia "transient global amnesia". J Neurol Neurosurg Psychiatry. 1966;29(5):472-474.

12. Bender MB. Syndrome of isolated episode of confusion with amnesia. J Hillside Hosp. 1956;5:212-215.

13. Fisher CM, Adams RD. Transient global amnesia. Trans Am Neurol Assoc. 1958;83:143-146.

14. Fisher CM, Adams RD. Transient global amnesia. Acta Neurol Scand Suppl. 1964;40(suppl 9):1-83.

15. Hodges JR, Warlow CP. Syndromes of transient amnesia: towards a classification. A study of 153 cases. J Neurol Neurosurg Psychiatry. 1990;53(10):834-843.

16. Kitamura T, Ogawa S, Roy DS, et al. Engrams and Circuits crucial for systems consolidation of a memory. Science. 2017;356(6333): 73-78.

17. Lee HY, Kim JH, Weon YC, et al. Diffusion-weighted imaging in transient global amnesia exposes the CA1 region of the hippocampus. Neuroradiology. 2007;49(6):481-487.

18. Bartsch T, Deuschl G. Transient global amnesia: functional anatomy and clinical implications. Lancet Neurol. 2010;9(2):205-214.

19. Bartsch T, Alfke K, Stingele R, et al. Selective affection of hippocampal CA-1 neurons in patients with transient global amnesia without longterm sequelae. Brain. 2006;129(pt 11):2874-2884.

20. Sander K, Sander D. New insights into transient global amnesia: recent imaging and clinical findings. Lancet Neurol. 2005;4(7):437-444.

21. Förster A, Griebe M, Gass A, Kern R, Hennerici MG, Szabo K. Diffusion-Weighted imaging for the differential diagnosis of disorders affecting the hippocampus. Cerebrovasc Dis. 2012;33(2):104-115.

22. Xing C, Arai K, Lo EH, et al. Pathophysiologic cascades in ischemic stroke. Int J Stroke. 2012;7(5):378-385. 
23. Peer M, Nitzan M, Goldberg I, et al. Reversible functional connectivity disturbances during Transient Global Amnesia. Ann Neurol. 2014;75(5): 634-643.

24. Saito K, Kimura K, Minematsu K, et al. Transient global amnesia assocaiated with an acute infaction in the retrosplenium of the corpus callosum. J Neurol Sci. 2003;210(1-2):95-97.

25. Ravindran V, Jain S, Ming A, Bartlett RJ. Transient global amnesia in a patient with acute unilateral caudate nucleus ischemia. J Clin Neurosci. 2004;11(6):669-672.

26. Yoon B, Yoo JY, Shim YS, et al. Transient Global Amnesia associated with acute intracerebral hemorrhage at the cingulate gyrus. Eur Neurol. 2006;56(1):54-56.

27. Graff-Radford J, Clapp AJ, Lanzino G, et al. Transient amnesia after coiling of a posterior circulation aneurysm. Neurocrit Care. 2013;18(2): 245-247.

28. Spiegel DR, Mccroskey AL, Deyerle BA. A case of transient global amnesia: a review and how it may shed further insight into the neurobiology of delusions. Innov Clin Neurosci. 2016;13(3-4):32-41.

29. Kimura K, Minematsu K, Yasaka M, Wada K, Yamaguchi T. The duration of symptoms in transient ischemic attack. Neurology. 1999;52(5): 976-980.

30. Lewis SL. Aetiology of transient global amnesia. Lancet. 1998;352(9125): 397-399.

31. Klötzsch C, Sliwka U, Berlit P, Noth J. An increased frequency of patent foramen ovale in patients with transient global amnesia: analysis of 53 consecutive patients. Arch Neurol. 1996;53(6):504-508.

32. De Francisco J, Pujadas F, Toledo M, et al. A study of right-left shunt in transient global amnesia. Neurologia. 2010;25(2):83-89.

33. Pantoni L, Lamassa M, Inzitari D. Transient global amnesia: a review emphasizing pathogenic aspects. Acta Neurol Scand. 2000;102(5): 275-283.

34. Schmidtke K, Reinhardt M, Krause T. Cerebral perfusion during transient global amnesia: findings with HM-PAO SPECT. J Nucl Med. 1998;39(1):155-159.

35. Winbeck K, Etgen T, von Einsiedel HG, Röttinger M, Sander D. DWI in transient global amnesia and TIA: proposal for an ischaemic origin of TGA. J Neurol Neurosurg Psychiatry. 2005;76(3):438-441.

36. Yang Y, Kim S, Kim JH. Ischemic evidence of transient global amnesia: location of the lesion in the hippocampus. J Clin Neurol. 2008;4(2): 59-66.

37. Di Filippo M, Calabresi P. Ischemic bilateral hippocampal dysfunction during transient global amnesia. Neurology. 2007;69(5):493.

38. Nakada T, Kwee IL, Fujii Y, et al. High-field, T2 reversed MRI of the hippocampus in transient global amnesia. Neurology. 2005;64(7): 1170-1174.

39. Huber R, Aschoff AJ, Ludolph AC, et al. Transient global amnesia. Evidence against vascular ischemic etiology from diffusion weighted imaging. J Neurol. 2002;249(11):1520-1524.

40. Chung CP, Hsu HY, Chao AC, et al. Transient global amnesia: cerebral venous outflow impairment-insight from the abnormal flow patterns of the internal jugular vein. Ultrasound Med Biol. 2007;33(11):1727-1735.

41. Cejas C, Cisneros LF, Lagos R, Zuk C, Ameriso SF. Internal jugular vein valve incompetence is highly prevalent in transient global amnesia. Stroke. 2010;41(1):67-71.

42. Baracchini C, Tonello S, Farina F, et al. Jugular veins in transient global amnesia: innocent bystanders. Stroke. 2012;43(9):2289-2292.

43. Faust JS, Nemes A. Transient global amnesia: emergency department evaluation and management. Emerg Med Pract. 2016;18(6):1-20.

44. Kang Y, Kim E, Kim JH, et al. Time of flight MR angiography assessment casts doubt on the association between transient global amnesia and intracranial jugular venous reflux. Eur Radiol. 2015;25(3): 703-709.

45. Owen D, Paranandi B, Sivakumar R, Seevaratnam M. Classical diseases revisited: transient global amnesia. Postgrad Med J. 2007;83(978): 236-239.

46. Olsen TS. Pathophysiology of the migraine aura: the spreading depression theory. Brain. 1995;118(pt 1):307-308.
47. Olesen J, Jørgensen MB. Leao's spreading depression in the hippocampus explains transient global amnesia: a hypothesis. Acta Neurol Scand. 1986; 73(2):219-220

48. Zeman AZ, Boniface SJ, Hodges JR. Transient epileptic amnesia: a description of the clinical and neuropsychological features in 10 cases and a review of the literature. JNeurol Neurosurg Psychiatry. 1998;64(4): 435-443.

49. Kapur N. Transient epileptic amnesia-a clinical update and a reformulation. J Neurol Neurosurg Psychiatry. 1993;56(11):1184-1190.

50. Serafetinides EA. Transient epileptic amnesia-a clinical update and a reformulation. J Neurol Neurosurg Psychiatry. 1994;57(12):1549.

51. Donnet A. Transient global amnesia triggered by migraine in a French Tertiary-Care Center: an 11-year retrospective analysis. Headache. 2015;55(6):853-859.

52. Bilo L, Meo R, Ruosi P, de Leva MF, Striano S. Transient epileptic amnesia: an emerging late-onset epileptic syndrome. Epilepsia. 2009; 50(suppl 5):58-61.

53. Butler CR, Zeman AZ. Recent insights into the impairment of memory in epilepsy: transient epileptic amnesia, accelerated long-term forgetting and remote memory impairment. Brain. 2008;131(pt 9):2243-2263.

54. Hoefeijzers S, Dewar M, Della Sala S, Zeman A, Butler C. Accelerated long-term forgetting in transient epileptic amnesia: an acquisition or consolidation deficit? Neuropsychologia. 2013;51(8):1549-1555.

55. Pérez-Lázaro C, Santos S, Garcés-Redondo M, et al. Amnesic stroke caused by hippocampal infarction. Rev Neurol. 2005;41(1):27-30.

56. Carlesimo GA, Lombardi MG, Caltagirone C. Vascular thalamic amnesia: a reappraisal. Neuropsychologia. 2011;49(5):777-789.

57. Adler AC, Warum D, Sapire JM. Transient global amnesia caused by hippocampal infarct: case report and review of literature. Clin Imaging. 2012;36(5):584-586

58. Liang JF, Shen AL, Lin SK. Bilateral hippocampal abnormalities on diffusion-weighted MRI in transient global amnesia: report of a case. Acta Neurol Taiwan. 2009;18(2):127-129.

59. Greer DM, Schaefer PW, Schwamm LH. Unilateral temporal lobe stroke causing ischemic transient global amnesia: role for diffusionweighted imaging in the initial evaluation. JNeuroimaging. 2001;11(3): 317-319.

60. Gupta M, Kantor MA, Tung CE, Zhang N, Albers GW. Transient global amnesia associated with a unilateral infarction of the fornix: case report and review of the literature. Front Neurol. 2015;5:291.

61. Li J, Hu WL. Bilateral hippocampal abnormalities in magnetic resonance imaging in transient global amnesia. Am J Emerg Med. 2013;31(4): 755.e1-e3.

62. Sedlaczek O, Hirsch JG, Grips E, et al. Detection of delayed focal MR changes in lateral hippocampus in transient global amnesia. Neurology. 2004;62(12):2165-2170.

63. Alemdar M, Selekler M. Migraine and cortical spreading depression. Agri. 2006;18(4):24-30.

64. Tosi L, Righetti CA. Transient global amnesia and migraine in young people. Clin Neurol Neurosurg. 1997;99(1):63-65.

65. Saito Y, Memezawa H. Migraine with aura accompanied by transient global amnesia. No To Hattatsu. 2010;42(4):303-304.

66. Maggioni F, Mainardi F, Bellamio M, Zanchin G. Transient global amnesia triggered by migraine in monozygotic twins. Headache. 2011; 51(8):1305-1308.

67. Romero JR, Mercado M, Beiser AS, et al. Transient global amnesia and neurological events: the framingham heart study. Front Neurol. 2013; 14(4):47.

68. Dalla Volta G, Zavarise P, Ngonga G, et al. Transient global amnesia as a presenting aura. Headache. 2014;54(3):551-552.

69. Ferlazzo E, Italiano D, Belcastro V, et al. Transient global amnesia as a presenting aura or epilepsy? Headache. 2014;54(7):1233-1235.

70. Schmidtke K, Ehmsen L. Transient global amnesia and migraine. A case control study. Eur Neurol. 1998;40(1):9-14.

71. Kumral E, Deveci EE, Erdoğan C, Enüstün C. Isolated hippocampal infarcts: vascular and neuropsychological findings. J Neurol Sci. 2015; 356(1-2):83-89. 
72. Blumenfeld AE, Victorio MC, Berenson FR. Complicated migraines. Semin Pediatr Neurol. 2016;23(1):18-22.

73. Jackson A, Stewart G, Wood A, Gillespie JE. Transient global amnesia and cortical blindness after vertebral angiography: further evidence for the role of arterial spasm. AJNR Am J Neuroradiol. 1995; 16(4 suppl):955-959.

74. Yildiz A, Yencilek E, Apaydin FD, Duce MN, Ozer C, Atalay A. Transient partial amnesia complicating cardiac and peripheral arteriography with nonionic contrast medium. Eur Radiol. 2003;13(suppl 4): L113-L115.

75. Benke T, Chemelli A, Lottersberger C, Waldenberger P, Karner E, Trinka E. Transient global amnesia triggered by the intracarotidamobarbital procedure. Epilepsy Behav. 2005;6(2):274-278.

76. Kim HY, Kang HS, Roh HG, et al. Transient global amnesia following vertebral artery angioplasty and stenting. Eur Neurol. 2006; 56(2):133-135.

77. Kaveeshvar H, Kashouty R, Loomba V, Yono N. A rare case of aortic dissection presenting as pure transient global amnesia. Cardiovasc $J$ Afr. 2015;26(6):e8-e9.

78. April MD, Fossum K, Hounshell C, et al. A sinister cause of anterograde amnesia: painless aortic dissection. Am J Emerg Med. 2015;33(7):989. e5-e7.

79. Irioka T, Yamanami A, Yagi Y, Mizusawa H. Aortic dissection as a possible cause of pure transient global amnesia: a case report and literature review. Neurol Sci. 2009;30(3):255-258.

80. Bonnet $\mathrm{P}, \mathrm{Niclot} \mathrm{P}, \mathrm{Chaussin} \mathrm{F}$, et al. A puzzling case of transient global amnesia. Lancet. 2004;364(9433):554.

81. Colotto M, Maranghi M, Epifania A, et al. Unmasking aortic dissection in patients of transient global amnesia: case report and diagnostic algorithm for the emergency department. BMJ Case Rep. 2011; 2011:bcr0720103151.

82. Machado A, Rodrigues M, Ribeiro M, et al. Tadalafil-induced transient global amnesia. J Neuropsychiatry Clin Neurosci. 2010;22(3): 352.e28.

83. Inzitari D, Pantoni L, Lamassa M, Pallanti S, Pracucci G, Marini P. Emotional arousal and phobia in transient global amnesia. Arch Neurol. 1997;54(7):866-873.

84. Neri M, Andermarcher E, De Vreese LP, Rubichi S, Sacchet C, Cipolli C. Transient global amnesia: memory and metamemory. Aging (Milano). 1995;7(6):423-429.

85. Zorzon M, Antonutti L, Masè G, Biasutti E, Vitrani B, Cazzato G. Transient global amnesia and transient ischemic attack: natural history, vascular risk factors, and associated conditions. Stroke. 1995;26(9):1536-1542.

86. Fisher CM. Transient global amnesia. Precipitating activities and other observations. Arch Neurol. 1982;39(10):605-608.

87. Miller JW, Petersen RC, Metter EJ, et al. Transient global amnesia: clinical characteristics and prognosis. Neurology. 1987;37(5):733-737.

88. Madias JE. Transient global amnesia and Takotsubo syndrome: would cerebral blood flow brain scan be of any help? Clin Auton Res. 2015;25(3):199.

89. Finsterer J, Aliyev R. Takotsubo syndrome: consequence or cause of ischemic stroke. Funct Neurol. 2014;29(4):281-282.

90. Finsterer J, Wahbi K. CNS disease triggered by Takotusbo stress cardiomyopathy. Int J Cardiol. 2014;177(2):322-329.

91. Abi-Saleh B, Iskandar SB, Schoondyke JW, et al. Tako-Tsubo syndrome as consequence of transient ischemic attack. Rev Cardiovasc Med. 2006;7(1):37-41.

Neuropsychiatric Disease and Treatment

\section{Publish your work in this journal}

Neuropsychiatric Disease and Treatment is an international, peerreviewed journal of clinical therapeutics and pharmacology focusing on concise rapid reporting of clinical or pre-clinical studies on a range of neuropsychiatric and neurological disorders. This journal is indexed on PubMed Central, the 'PsycINFO' database and CAS,
92. Lyon AR, Rees PS, Prasad S, et al. Stress (Takotsubo) cardiomyopathy-a novel pathophysiological hypothesis to explain catecholamine-induced acute myocardial stunning. Nat Clin Pract Cardiovasc Med. 2008;5(1): 22-29.

93. Finsterer J, Stöllberger C. Transient global amnesia: the cerebral Takotsubo? J Neurol Sci. 2017;15(376):196-197.

94. Jäger T, Bäzner H, Kliegel M, Szabo K, Hennerici MG. The transience and nature of cognitive impairments in transient global amnesia: a meta-analysis. J Clin Exp Neuropsychol. 2009;31(1):8-19.

95. Uttner I, Prexl S, Freund W, Unrath A, Bengel D, Huber R. Long-term outcome in transient global amnesia patients with and without focal hyperintensities in the CA1 region of the hippocampus. Eur Neurol. 2012;67(3): 155-160.

96. Noël A, Quinette P, Dayan J, et al. Influence of patients' emotional state on the recovery processes after a transient global amnesia. Cortex. 2011;47(8):981-991.

97. Mangla A, Navi BB, Layton K, et al. Transient global amnesia and the risk of ischemic stroke. Stroke. 2014;45(2):389-393.

98. Brown J. ED evaluation of transient global amnesia. Ann Emerg Med. 1997;30(4):522-526.

99. Strupp M, Brüning R, Wu RH, Deimling M, Reiser M, Brandt T. Diffusion-weighted MRI in transient global amnesia: elevated signal intensity in the left mesial temporal lobe in 7 of 10 patients. Ann Neurol. 1998;43(2):164-170.

100. Schaefer PW. Diffusion-weighted imaging as a problem solving tool in the evaluation of patients with acute stroke-like syndromes. Top Magn Reson Imaging. 2000;11(5):300-309.

101. Tiecks FP, Lam AM, Matta BF, Strebel S, Douville C, Newell DW. Effects of the Valsalva maneuver on cerebral circulation in healthy adults. A transcranial Doppler study. Stroke. 1995;26(8):1386-1392.

102. Tchalla AE, Marin B, Mignard C, et al. Newly diagnosed epileptic seizures: focus on an elderly population on the French island of Reunion in the Southern Indian Ocean. Epileptic Disord. 2013;15(3):243-254.

103. Melo TP, Ferro JM, Ferro H. Transient global amnesia. A case control study. Brain. 1992;115(pt 1):261-270.

104. Guidotti M, Anzalone N, Morabito A, Landi G. A case-control study of transient global amnesia. J Neurol Neurosurg Psychiatry. 1989;52(3): 320-323.

105. Bartsch T, Butler C. Transient amnesic syndromes. Nat Rev Neurol.2013; 9(2):86-97.

106. Sánchez-Porras R, Zheng Z, Sakowitz OW. Pharmacological modulation of spreading depolarizations. Acta Neurochir Suppl. 2015;120: 153-157.

107. Kurdi MS, Theerth KA, Deva RS. Ketamine: current applications in anesthesia, pain, and critical care. Anesth Essays Res. 2014;8(3): 283-290.

108. Ryoo I, Kim JH, Kim S, Choi BS, Jung C, Hwang SI. Lesion detectability on diffusion-weighted imaging in transient global amnesia: the influence of imaging timing and magnetic field strength. Neuroradiology. 2012;54(4):329-334.

109. Arena JE, Rabinstein AA. Transient global amnesia. Mayo Clin Proc. 2015;90(2):264.

110. Williamson J, Larner AJ. Transient global amnesia. Br J Hosp Med (Lond). 2015;76(12):C186-C188.

111. Kaveeshvar H, Kashouty R, Loomba V. et al. A rare case of aortic dissection presenting as pure transient global amnesia. Cardiovasc J Afr. 2015;26(6):e8-e9. 